\title{
Low birth weight and risk of affective disorders and selected medical illness in offspring at high and low risk for depression
}

\author{
Yoko Nomura $^{\mathrm{a}, *}$, Priya J. Wickramaratne ${ }^{\mathrm{b}, \mathrm{d}}$, Daniel J. Pilowsky ${ }^{\mathrm{b}, \mathrm{c}, \mathrm{e}}$, Jeffrey H. Newcorn ${ }^{\mathrm{a}}$, \\ Beth Bruder-Costello ${ }^{\mathrm{b}, \mathrm{e}}$, Charles Davey ${ }^{\mathrm{h}}$, William P. Fifer ${ }^{\mathrm{b}, \mathrm{e}, \mathrm{f}}$, \\ Jeanne Brooks-Gunn ${ }^{\mathrm{f}, \mathrm{g}}$, Myrna M. Weissman ${ }^{\mathrm{b}, \mathrm{c}, \mathrm{e}}$ \\ ${ }^{\mathrm{a}}$ Department of Psychiatry, Mount Sinai School of Medicine, New York, NY 10029, USA \\ ${ }^{\mathrm{b}}$ New York State Psychiatric Institute, New York, NY 10032, USA \\ ${ }^{\mathrm{c}}$ Department of Epidemiology, Columbia University School of Public Health, New York, NY 10032, USA \\ ${ }^{\mathrm{d}}$ Department of Biostatistics, Columbia University School of Public Health, New York, NY 10032, USA \\ ${ }^{\mathrm{e}}$ Department of Psychiatry, Columbia University College of Physicians and Surgeons, New York, NY 10032, USA \\ ${ }^{\mathrm{f}}$ Department of Pediatrics, Columbia University College of Physicians and Surgeons, New York, NY 10032, USA \\ ${ }^{\mathrm{g}}$ Columbia University Teachers College, New York, NY 10027, USA \\ ${ }^{\mathrm{h}}$ The School, Columbia University, New York, NY 10025, USA
}

\begin{abstract}
Numerous studies have demonstrated that low birth weight (LBW) is associated with the development of medical conditions, such as hypertension and diabetes, and psychiatric disorders, such as depression. One possible mechanism through which LBW might increase risk for both medical and psychiatric disorders is by altering the biologic systems (such as the hypothalamic-pituitary-adrenal [HPA] axis function) that govern emotion regulation and physical reactivity. In this study, we conducted secondary data analyses in a longitudinal study originally designed to understand the intergenerational transmission of major depressive disorder (MDD). We examined the risk for both medical and psychiatric illnesses known to be influenced by HPA axis dysregulation in the context of parental depression. The study had 2 primary objectives: (1) to examine whether LBW increases the risk of selected adult illness that may be influenced by the HPA axis and (2) to examine whether the increased risk of illness varies by parental depression status.

We conducted longitudinal assessments of 244 offspring of depressed and nondepressed parents for more than 20 years. Psychopathology and medical illness were assessed by direct interview conducted by clinicians blind to risk status and previous diagnosis. We examined the effect of BW in 3 categories: less than $2.5 \mathrm{~kg}$ (LBW), 2.5-3.5 kg, and more than $3.5 \mathrm{~kg}$ (reference group).

Offspring with LBW had a significantly increased risk of MDD, anxiety disorders, phobia, suicidal ideation, impaired functioning, allergies, and hypertension compared to those with BW exceeding $3.5 \mathrm{~kg}$. The association between LBW and depression was stronger among children of depressed parents than among children of nondepressed parents, with an interaction term (BW and parental depression status) significant for MDD $(P=.05)$, suggesting that parental depression may augment the impact of LBW on offspring depression:
\end{abstract} (C) 2007 Elsevier Inc. All rights reserved.

The study received support in the form of R01MH036197 (PI, Myrna M. Weissman) from the NIMH, Young Investigator Award (PI, Yoko Nomura) from the National Alliance for Research on Schizophrenia and Depression (NARSAD), R03 MH 067761 (PI, Yoko Nomura) from the NIMH, the Sackler Institute for Developmental Psychobiology, and Child and Families Resilience Program at Mount Sinai School of Medicine (Director, Claude M. Chemtob).

* Corresponding author. Department of Psychiatry, Mount Sinai School of Medicine, One Gustave L. Levy Pl, Box 1230, New York, NY 100296574, USA. Tel.: +1 212987 0335; fax: +1 2129870117.

E-mail address: yoko.nomura@mssm.edu (Y. Nomura).

\section{Introduction}

Low birth weight (LBW), defined as birth weight (BW) of less than $2.5 \mathrm{~kg}$, may increase the risk of medical and psychiatric problems across the life cycle. School-aged children born with LBW are vulnerable to behavioral [1], emotional [2], and medical problems [3], poor cognitive functioning [4], and learning difficulties [4,5]. Barker's fetal origins hypothesis [6] also suggests a link between LBW and adult hypertension, stroke [7], and diabetes mellitus [8]. Furthermore, recent studies showed that LBW increased the 
risk of psychopathology, such as emotional distress and depression in adolescents and adults [9-12].

Psychosocial stress in pregnancy [13] may contribute to decreased BW through increases in hypothalamic corticotropin-releasing hormone $(\mathrm{CRH})$ release [14]. Exposure to stress in utero may also underlie vulnerability to subsequent normative stress and biophysiologic changes in the hypothalamic-pituitary-adrenal (HPA) axis. Taken together, having LBW may compromise the integrity of the HPA axis functionality [15], which may be associated with emotional disorders and behavioral inhibition as well as medical illnesses related to cardiovascular reactivity and respiratory, and immune functionality [16]. The present study examined whether the effect of LBW was found in other illnesses associated with HPA axis functionality such as depression, anxiety disorders, hypertension, allergies and respiratory illness [17] as well as global functioning of the offspring. We further examined whether the risk was greater if concomitant with parental depression, which might place offspring with LBW at increased risk for disorders from familial and environmental factors [18].

\section{Methods}

\subsection{Subjects}

The study sample consisted of offspring of depressed and nondepressed probands participating in an ongoing longitudinal study of the impact of parental depression on the offspring. Depressed probands were recruited from a treatment center and nondepressed probands were recruited from the same community where the treatment center was located. The depressed and nondepressed probands were group-matched by age and sex (see [19-22]). After the initial assessment, 244 offspring with information on BW were interviewed 4 times over a 20 -year period and followed into adulthood (mean age, 33 years; SD, 8.8). Response rates were $80 \%$ or higher at each wave of data collection. Nonresponse rates did not vary by proband depression status or BW. Of these 244 offspring, 162 were offspring of depressed parents (defined as either parent depressed); 82 were offspring of nondepressed parents; $8.2 \%$ of offspring had LBW $(<2.5 \mathrm{~kg}), 54.5 \%$ had BW between 2.5 and $3.5 \mathrm{~kg}$, and $37.3 \%$ had BW of more than $3.5 \mathrm{~kg}$. All interview waves were approved by the institutional review board at New York State Psychiatric Institute/Columbia University. After a complete description of the study was provided to the subjects, written informed consent was obtained from adults and assent was obtained from minors who also had written consent from their parents.

\subsection{Assessments}

Psychiatric diagnosis was obtained through the Schedule for Affective Disorders and Schizophrenia-Lifetime Version for adults [23] and a slightly modified version of the Schedule for Affective Disorders and Schizophrenia for School-Aged
Children, Epidemiologic Version [24] for ages 6 to 17. The diagnostic assessments were administered by trained doctoral and masteral level mental health professionals, who were blind to the clinical status of the parents and to previous history. Multiple sources of information were used, including direct and informant interviews and medical records wherever available. The Global Assessment Scale (GAS) was completed at each wave [25]. This instrument is rated on a 100 -point scale and provides an overall estimate of the person's current functional adjustment. Another version for children, the C-GAS [26], was used when the offspring were between ages 6 and 17. Lower scores on the GAS or C-GAS indicated more overall impairment. Diagnoses, GAS, and episodes of suicidal ideation were based on the best-estimate procedure, which is described elsewhere [22,27]. All diagnoses for the offspring were cumulative across all waves.

Information on medical illness in offspring was collected at each wave. Using a checklist format, either the parent or the offspring (depending on age) indicated "yes" or "no" to both a lifetime and current history of medical problems. The age at first onset of each medical problem was ascertained as well. All ambiguous reports of medical problems, including any discrepancy between medical charts obtained from their physicians and the interview, were recoded by a physician blind to the depression status of the offspring and parents. Information on each medical illness was pooled to create cumulative variables indicating a lifetime history of medical conditions.

Birth weight was extracted from parents' report of the child's developmental history at the baseline assessment, except for 17 cases $(9 \%)$ whose BW was collected again at a later assessment. Although a very small proportion, the correlation between the 2 reports on BW was high $(r=.87)$. Birth weight was divided into 3 categories: $<2.5,2.5-3.5$, $>3.5 \mathrm{~kg}$. We refer to BW less than $2.5 \mathrm{~kg}$ as "LBW," BW between 2.5 and $3.5 \mathrm{~kg}$ as "mid BW," and BW greater than $3.5 \mathrm{~kg}$ as "high BW" throughout the article. Normal BW refers to values exceeding $2.5 \mathrm{~kg}$ (mid and high $\mathrm{BW}$ ). Information on maternal smoking, drinking, and substance use during pregnancy was also gathered from parents' report (mostly mothers). Information on socioeconomic status (SES) was determined with the Hollingshead 4-factor index, which incorporates the education and occupation of mothers and fathers [28].

\subsection{Data analysis}

To examine differences in rates of disorders in offspring among the $3 \mathrm{BW}$ groups $(<2.5,2.5-3.5,>3.5 \mathrm{~kg})$, univariate analysis was conducted by using $\chi^{2}$ tests. The same analysis was repeated after stratification by parental depression status.

The univariate analyses were followed by multivariate analyses to adjust for potential confounders: the Cox proportional hazard regression model was used to adjust for the differences in follow-up time for each offspring [29]. Sex and preterm birth status of offspring, parental depression, SES, mother's smoking, alcohol, and drug use during 
pregnancy, parity, and maternal age at the birth of the offspring were considered a priori as potential confounders, and were included in the model for statistical adjustment. Age of offspring was not included in the model as a covariate because Cox proportional hazard analyses implicitly adjusts for differences in age at follow-up (ie, age at last interview). We used an average of CGAS or GAS scores across waves as a measure of global functioning, because it reflects functioning from childhood to early adulthood. We used a cutoff score of 65 or less as impaired functioning, the suggested cutoff for children and adolescents [30]. Logistic regression analyses were used to examine the effect of BW on the offspring's global functioning, because the functioning measure was averaged across waves. The product term of BW $(<2.5,2.5-3.5,>3.5 \mathrm{~kg})$ and parental depression measures was added for testing possible interaction effect. The same set of potential confounders was included in this model as covariates, and age of offspring was added as a covariate. Lastly, lifetable analyses were conducted to explore differences in age-specific rates of major depressive disorder (MDD), anxiety disorders, phobia, and any medical illness by BW status. We used dichotomous BW (LBW vs normal $\mathrm{BW}$ ), instead of the 3 categories, as patterns of agespecific incidence for the 2 normal BW groups (mid BW and high $\mathrm{BW}$ ) were similar. Because no formal tests of differences in patterns were performed, these analyses should be viewed as descriptive.

The study allowed for the inclusion of more than 1 offspring from the same family. Consequently, the assumption of independence of the outcome variable implicit in the use of the standard Cox proportional hazard model may be violated. To overcome this problem, we used the methods of Binder [31], who extended the methods of Lin and Wei [32]. They proposed a method for estimating the covariance matrix of the estimated parameters when the model is misspecified in those situations where there is correlation among sample units. We used SUDAAN to obtain the adjusted variance for the relevant parameters [33].

\section{Results}

\subsection{Characteristics of offspring and their mothers}

There were no significant differences among the $3 \mathrm{BW}$ groups $(<2.5,2.5-3.5,>3.5 \mathrm{~kg})$ on any major demographic variable, except for age of offspring. Mean (SD) age of offspring with LBW, mid BW (2.5-3.5 kg), and high BW $(>3.5 \mathrm{~kg})$ was $36.3(5.7), 33.1$ (7.8), and 30.5 (10.2), respectively $\left(\mathrm{F}_{2,241}=4.8, P=.009\right)$. Fifty-five percent of the offspring were female, $51 \%$ were married, $73 \%$ Catholic, $16 \%$ Protestant, and $11 \%$ other religions. Mean (SD) family and individual incomes were \$64574 (28053) and \$39608 (22 646), respectively.

With regard to mothers' characteristics that may be associated with $\mathrm{BW}$, a little over half $(58.6 \%)$ of the mothers did not smoke at all, $82 \%$ did not imbibe any alcoholic beverages, and almost all (99\%) did not use any illicit drug during pregnancy. Although the rate of maternal drinking and substance use during pregnancy did not differ among the $3 \mathrm{BW}$ groups, the rate of maternal smoking during pregnancy (ever smoked) was significantly different among the 3 groups: $60 \%$ of mothers of LBW, $36 \%$ of mothers of mid BW, and $20 \%$ of mothers of high BW offspring smoked during pregnancy, respectively $(P=.001)$. Half of the LBW offspring were born preterm, and approximately $8 \%$ of mid and high BW offspring were born preterm $(P<$ $.0001)$. There was no difference in maternal age at birth (mean, 27.2; SD, 5.6) and parity (mean, 2.4; SD, 1.4) among the $3 \mathrm{BW}$ groups.

\subsection{Cumulative rates of offspring disorders by $B W$}

We examined the rate of each offspring disorder according to $\mathrm{BW}$. We began by evaluating the overall differences in the rates of each disorder (both psychiatric and medical) among the $3 \mathrm{BW}$ groups. As shown in Table 1, the rate of disorder for LBW was substantially higher than that for high BW offspring, for example, MDD ( $75 \%$ vs $32 \%$ ), any anxiety disorder $(65 \%$ vs $36 \%)$, phobia ( $50 \%$ vs $19 \%$ ), suicidal ideation ( $40 \%$ vs $11 \%$ ), impaired functioning ( $45 \%$ vs $24 \%$ ), respiratory illness $(44 \%$ vs $19 \%)$, hypertension ( $15 \%$ vs $4 \%$ ), and allergies (67\% vs $36 \%$ ). The cumulative rate of disorders for offspring with mid BW fell in between that of LBW and high BW. Overall group differences for the rate of offspring disorders among the $3 \mathrm{BW}$ groups were significant, for all outcome variables except impaired functioning and hypertension.

Table 1

Rates per 100 (SE) of psychiatric and medical problems in offspring according to $\mathrm{BW}$

\begin{tabular}{|c|c|c|c|c|c|c|}
\hline \multirow[t]{2}{*}{$\begin{array}{l}\text { Offspring } \\
\text { disorders }\end{array}$} & \multicolumn{3}{|c|}{ Offspring BW statistics } & \multicolumn{3}{|c|}{$\begin{array}{c}\text { Overall } \\
\text { difference }\end{array}$} \\
\hline & $\begin{array}{l}<2.5 \mathrm{~kg} \\
(\mathrm{n}=20), \\
\%(\mathrm{SE})\end{array}$ & $\begin{array}{l}2.5-3.5 \mathrm{~kg} \\
(\mathrm{n}=133), \\
\%(\mathrm{SE})\end{array}$ & $\begin{array}{l}>3.5 \mathrm{~kg} \\
(\mathrm{n}=91) \\
\%(\mathrm{SE})\end{array}$ & $\chi^{2}$ & $d f$ & $P$ \\
\hline MDD & 75.0 (9.9) & $47.4(4.3)$ & $31.9(4.9)$ & 13.9 & 2 & .001 \\
\hline $\begin{array}{l}\text { Any anxiety } \\
\text { disorder }\end{array}$ & $65.0(10.9)$ & $44.4(4.3)$ & $36.3(5.1)$ & 5.8 & 2 & .05 \\
\hline Any phobia & $50.0(11.5)$ & $24.1(3.7)$ & $18.7(4.1)$ & 8.8 & 2 & .01 \\
\hline $\begin{array}{l}\text { Suicidal } \\
\text { ideation }\end{array}$ & $40.0(11.2)$ & $18.0(3.3)$ & $11.0(3.3)$ & 9.8 & 2 & .007 \\
\hline $\begin{array}{l}\text { Impaired } \\
\text { functioning }\end{array}$ & $45.0(11.4)$ & $27.1(3.9)$ & $24.1(4.6)$ & 3.6 & 2 & .17 \\
\hline $\begin{array}{l}\text { Respiratory } \\
\text { illness }\end{array}$ & $44.4(12.1)$ & $31.0(4.0)$ & $17.6(4.3)$ & 6.1 & 2 & .04 \\
\hline Hypertension & $15.0(8.2)$ & $7.8(2.2)$ & $3.6(2.0)$ & 3.6 & 2 & .17 \\
\hline Allergies & $66.7(11.4)$ & $52.7(4.5)$ & $36.3(5.5)$ & 8.7 & 2 & .01 \\
\hline
\end{tabular}

$\mathrm{n}$ may vary because of missing values. Any anxiety disorder includes separation anxiety disorder, overanxious disorder, generalized anxiety disorder, obsessive compulsive disorder, panic disorder, posttraumatic stress disorders (PTSD), and phobia; any phobia includes simple phobia, social phobia, and agoraphobia. Impaired functioning was based on overall functioning scores across. 
For impaired functioning and hypertension, although offspring with LBW had the highest rates, the 2 other groups (mid and high BW) had similar rates, contributing to the nonsignificant findings.

\subsection{Cumulative rates of offspring disorders by BW stratified by parental depression status}

We examined the rate of each offspring disorder according to BW (Table 2) stratified by parental depression status. Among offspring of depressed parents, the rate of MDD, any anxiety disorder, any phobia, suicidal ideation, impaired functioning, and allergy was significantly different among the $3 \mathrm{BW}$ groups. As compared to high BW (>3.5 kg) offspring, LBW offspring had substantially higher rates of MDD (81.3\% vs $32.8 \%)$, any anxiety disorder (75\% vs $41.8 \%$ ), phobia (56.3\% vs $22.4 \%$ ), suicidal ideation $(43.8 \%$ vs $13.4 \%$ ), impaired functioning (50\% vs $23.8 \%)$, and allergies (66.7\% vs $36.3 \%)$. In contrast, among offspring of nondepressed parents, there was no overall difference in the rate of any disorder.

\subsection{Risk of offspring disorders by $B W$}

Results from Cox proportional hazards analyses are presented in Table 3. Relative risk (RR) was used as a risk indicator for all variables except for impaired functioning, where odds ratio (OR) was used (see Section 2.3 for detail). Offspring who had LBW, relative to those with high BW, had a more than 4-fold increased risk of hypertension (RR, 4.5; 95\% CI, 0.8-25.2) and impaired global functioning (OR, 6.1; 95\% CI, 1.5-22.8), an approximately 3-fold increased risk of MDD (RR, 2.9; 95\% CI, 1.4-6.0), any anxiety disorder (RR, 3.0; 95\% CI, 1.4-6.7), phobias (RR, 3.1; 95\% CI, 1.2-8.0) and suicidal ideation (RR, 2.7; 95\% CI, 1.0-7.2), as well as a 2-fold increased risk of allergies (RR, 2.0; 95\% CI, 1.0-4.2). Offspring who had mid BW (2.5-3.5 kg), relative to those with high $\mathrm{BW}$, had an almost 2-fold increased risk of MDD (RR, 1.7; 95\% CI, 1.0-2.7) and allergies (RR, 1.65; 95\% CI, 1.0-2.6), after adjusting for potential confounder variables. There was no significant increased risk of respiratory illness for offspring with LBW relative to those with high $\mathrm{BW}$, after controlling for potential confounders.

The possible moderating effect of parental depression was explored and the results are presented in the last column of Table 3. There was a statistically significant interaction between parental depression and BW status on $\operatorname{MDD}(P=$ $.05)$ and a marginally significant effect on impaired functioning $(P=.098)$, indicating that the adverse impact of BW (low, mid, and high) on offspring's MDD and impaired functioning may be stronger in the offspring of

Table 2

Rates per 100 (SE) of psychiatric and medical problems in offspring according to BW by parental depression status

\begin{tabular}{|c|c|c|c|c|c|c|}
\hline \multicolumn{7}{|c|}{ Neither parent depressed } \\
\hline \multirow[t]{2}{*}{ Offspring disorders } & \multicolumn{3}{|c|}{ Offspring BW statistics } & \multicolumn{3}{|c|}{ Overall difference } \\
\hline & $\begin{array}{l}<2.5 \mathrm{~kg}(\mathrm{n}=4) \\
\%(\mathrm{SE})\end{array}$ & $\begin{array}{l}2.5-3.5 \mathrm{~kg}(\mathrm{n}=54) \\
\%(\mathrm{SE})\end{array}$ & $\begin{array}{l}>3.5 \mathrm{~kg}(\mathrm{n}=24), \\
\%(\mathrm{SE})\end{array}$ & $\chi^{2}$ & $d f$ & $P$ \\
\hline MDD & $50.0(28.9)$ & $24.1(5.9)$ & $29.2(9.5)$ & 1.4 & 2 & .50 \\
\hline Any anxiety disorder & $25.0(25.0)$ & $22.2(5.7)$ & $20.8(8.5)$ & 0.4 & 2 & .98 \\
\hline Any phobia & $25.0(25.0)$ & $9.3(3.6)$ & $8.3(4.2)$ & 1.1 & 2 & .57 \\
\hline Suicidal ideation & $25.0(25.0)$ & $7.4(3.6)$ & $4.2(4.2)$ & 2.2 & 2 & .38 \\
\hline Impaired functioning & $25.0(25.0)$ & $9.3(4.0)$ & $25.0(9.0)$ & 3.7 & 2 & .16 \\
\hline Respiratory illness & $75.0(25.0)$ & $0.8(6.5)$ & $21.7(8.8)$ & 4.6 & 2 & .10 \\
\hline Hypertension & $0(-)$ & $4.3(3.0)$ & $0(-)$ & 1.0 & 2 & .60 \\
\hline Allergies & $50.0(28.9)$ & $53.8(7.0)$ & $37.5(10.4)$ & 1.8 & 2 & .42 \\
\hline \multicolumn{7}{|c|}{$\geq 1$ parent depressed } \\
\hline \multirow[t]{2}{*}{ Offspring disorders } & \multicolumn{3}{|c|}{ Offspring BW statistics } & \multicolumn{3}{|c|}{ Overall difference } \\
\hline & $\begin{array}{l}<2.5 \mathrm{~kg}(\mathrm{n}=16), \\
\%(\mathrm{SE})\end{array}$ & $\begin{array}{l}2.5-3.5 \mathrm{~kg}(\mathrm{n}=79) \text {, } \\
\%(\mathrm{SE})\end{array}$ & $\begin{array}{l}>3.5 \mathrm{~kg}(\mathrm{n}=69), \\
\%(\mathrm{SE})\end{array}$ & $\chi^{2}$ & $d f$ & $P$ \\
\hline MDD & $81.3(10.1)$ & $63.3(5.5)$ & $32.8(5.8)$ & 19.4 & 2 & .0001 \\
\hline Any anxiety disorder & $75.0(11.2)$ & $59.5(5.6)$ & $41.8(6.1)$ & 7.8 & 2 & .02 \\
\hline Any phobia & $56.3(12.8)$ & $34.2(5.4)$ & $22.4(5.1)$ & 7.4 & 2 & .03 \\
\hline Suicidal ideation & $43.8(12.8)$ & $25.3(4.9)$ & $13.4(4.2)$ & 7.7 & 2 & .02 \\
\hline Impaired functioning & $50.0(12.9)$ & $39.2(5.5)$ & $23.8(5.4)$ & 5.7 & 2 & .058 \\
\hline Respiratory illness & $35.7(13.3)$ & $31.2(5.3)$ & $17.7(4.9)$ & 3.9 & 2 & .14 \\
\hline Hypertension & $18.8(10.1)$ & $7.9(3.5)$ & $4.9(3.3)$ & 3.4 & 2 & .19 \\
\hline Allergies & $66.7(12.5)$ & $52.7(5.7)$ & $36.3(6.3)$ & 7.5 & 2 & .02 \\
\hline
\end{tabular}

$\mathrm{n}$ may vary because of missing values. Any anxiety disorder includes separation anxiety disorder, overanxious disorder, generalized anxiety disorder, obsessive compulsive disorder, panic disorder, PTSD, and phobia; any phobia includes simple phobia, social phobia, and agoraphobia. Impaired functioning was based on overall functioning scores across. 
Table 3

Risk and 95\% confidence interval (CI) for lifetime psychiatric and medical illness according to BW (kg)

\begin{tabular}{|c|c|c|c|c|}
\hline Illness in offspring (BW, kg) & $\mathrm{n}$ & Unadjusted risk $(95 \% \mathrm{CI}), P$ & Adjusted risk $(95 \% \mathrm{CI}), P$ & Interaction $^{\text {a }}$ Wald $\chi^{2}, P$ \\
\hline \multicolumn{5}{|l|}{ MDD } \\
\hline$<2.50$ & 20 & $2.7(1.5,5.1), P=.0017$ & $2.9(1.4,6.1), P=.004$ & \multirow[t]{3}{*}{$3.7, P=.050$} \\
\hline $2.50-3.50$ & 133 & $1.7(1.1,2.7), P=.02$ & $1.7(1.0,2.7), P=.04$ & \\
\hline$>3.50$ & 91 & 1.0 & 1.0 & \\
\hline \multicolumn{5}{|l|}{ Any anxiety disorder } \\
\hline$<2.50$ & 20 & $2.2(1.2,4.2), P=.02$ & $3.0(1.4,6.7), P=.006$ & \multirow[t]{3}{*}{$1.2, P=.28$} \\
\hline $2.50-3.50$ & 133 & $1.4(.9,2.1), P=.14$ & $1.3(.8,2.1), P=.24$ & \\
\hline$>3.50$ & 91 & 1.0 & 1.0 & \\
\hline \multicolumn{5}{|l|}{ Any phobia } \\
\hline$<2.50$ & 20 & $2.9(1.3,6.3), P=.0085$ & $3.1(1.2,8.0), P=.02$ & \multirow[t]{3}{*}{$.09, P=.77$} \\
\hline $2.50-3.50$ & 133 & $1.4(.8,2.6), P=.24$ & $1.3(.7,2.4), P=.50$ & \\
\hline$>3.50$ & 91 & 1.0 & 1.0 & \\
\hline \multicolumn{5}{|l|}{ Suicidal ideation } \\
\hline$<2.50$ & 20 & $3.2(1.3,8.2), P=.01$ & $2.7(1.0,7.2), P=.05$ & \multirow[t]{3}{*}{$.07, P=.78$} \\
\hline $2.50-3.50$ & 133 & $1.5(.7,3.5), P=.32$ & $1.5(.7,3.3), P=.30$ & \\
\hline$>3.50$ & 91 & 1.0 & 1.0 & \\
\hline \multicolumn{5}{|l|}{ Impaired functioning } \\
\hline$<2.50$ & 20 & $2.6(.9,7.3), P=.06$ & $6.1(1.5,22.8), P=.008$ & \multirow[t]{3}{*}{$3.2, P=.098$} \\
\hline $2.50-3.50$ & 133 & $2.4(1.0,5.6), P=.05$ & $1.8(.8,3.8), P=.13$ & \\
\hline$>3.50$ & 87 & 1.0 & 1.0 & \\
\hline \multicolumn{5}{|l|}{ Respiratory illness } \\
\hline$<2.50$ & 18 & $2.3(1.0,5.4), P=.05$ & $1.8(.9,3.6), P=.11$ & \multirow[t]{3}{*}{$.24, P=.74$} \\
\hline $2.50-3.50$ & 129 & $1.5(.8,2.6), P=.19$ & $1.3(.7,4.4), P=.74$ & \\
\hline$>3.50$ & 85 & 1.0 & 1.0 & \\
\hline \multicolumn{5}{|l|}{ Hypertension } \\
\hline$<2.50$ & 20 & $3.4(.7,16.4), P=.12$ & $4.5(.80,25.2), P=.09$ & \multirow[t]{3}{*}{$.65, P=.42$} \\
\hline $2.50-3.50$ & 128 & $1.5(.2,16.6), P=.74$ & $2.0(.15,25.6), P=.59$ & \\
\hline$>3.50$ & 83 & 1.0 & 1.0 & \\
\hline \multicolumn{5}{|l|}{ Allergies } \\
\hline$<2.50$ & 18 & $2.0(1.1,3.9), P=.03$ & $2.0(1.0,4.2), P=.05$ & \multirow[t]{3}{*}{$.88, P=1.7$} \\
\hline $2.50-3.50$ & 129 & $1.6(1.0,2.4), P=.04$ & $1.6(1.0,2.6), P=.04$ & \\
\hline$>3.50$ & 84 & 1.0 & 1.0 & \\
\hline
\end{tabular}

Impaired functioning was based on overall functioning scores across waves and there is no age of onset. Therefore, logistic regression analysis was used and the risk reported here is based on odds ratio. For other diagnoses, proportional hazards model was used and the risk reported is based on hazards ratio. Adjusted risk $=$ adjusted for preterm birth, sex, family SES, maternal risk behavior during pregnancy (drinking, smoking, and drug use), parity, maternal age at the birth of the offspring, and parental lifetime depression status.

Any anxiety disorder includes separation anxiety disorder, overanxious disorder, generalized anxiety disorder, obsessive compulsive disorder, panic disorder, PTSD, and phobia; and any phobia includes simple phobia, social phobia, and agoraphobia.

${ }^{a}$ The interaction between birth weight $(<2.50,2.50-3.50,>3.50)$ and parental depression status.

depressed parents, compared to the offspring of nondepressed parents. No other interactions between parental depression and BW status on offspring disorders were statistically significant.

\subsection{Age-specific rates of MDD, anxiety disorders, phobia,} and any medical illness

Figs. 1-4 show the results of estimating age-specific rates using lifetable analyses for MDD, anxiety disorders, phobia, and any medical illness under examination by dichotomous BW status (LBW vs normal BW). Visual inspection suggests that there was a difference in rates between the 2 groups. Fig. 1 shows that the peak incidence of MDD occurred between ages 15 and 25, and it was higher in the offspring with LBW compared to offspring with normal BW. Figs. 2 and 3 show that the peak incidence of anxiety disorders and phobia was between ages 5 and 15, and it was higher in the offspring with LBW compared to offspring with normal BW.
Fig. 4 shows that the peak incidence of any medical illness occurs later in adulthood, especially after age 25 , where a greater difference in rates between offspring with LBW and normal BW was observed.

\section{Discussion}

Using a longitudinal design, we examined whether BW was associated with psychopathology, selected medical illness, and global functioning in young adulthood, and whether the severity of sequelae increased with decreasing BW. We further examined whether parental depression amplified the adverse effect of LBW on offspring disorders and functioning. The findings suggest a 2 - to 4-fold increased risk of MDD $(P=.004)$, anxiety disorders $(P=$ $.006)$, phobia $(P=.02)$, suicidal ideation $(P=.05)$, hypertension $(P=.09)$ and allergies $(P=.05)$, and a 6-fold increased risk of impaired global functioning $(P=.008)$ in 


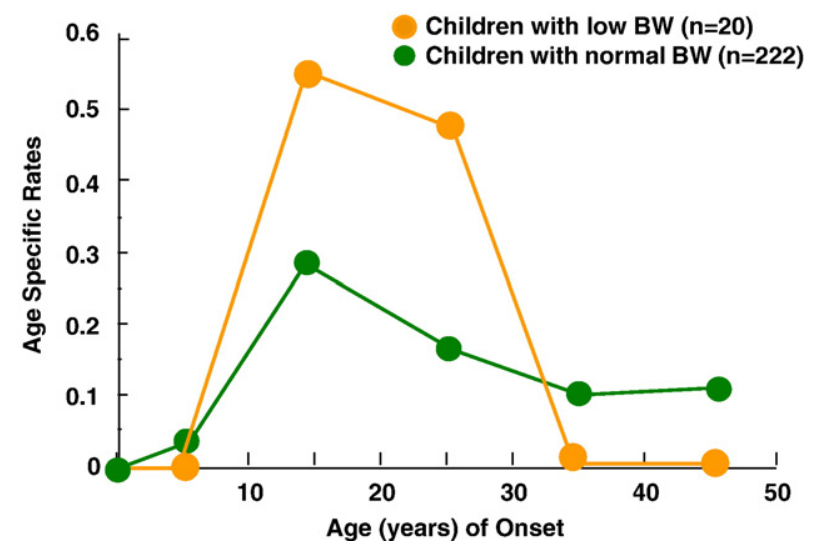

Fig. 1. Age-specific rates of major depression in children (second generation) by their low BW status.

LBW offspring compared to those with high BW. Furthermore, the interaction between parental depression and offspring BW status on offspring MDD was significant $(P=.05)$, suggesting that parental MDD amplified the effect of BW on offspring MDD. Notably, among offspring of depressed parents, $81 \%$ of LBW offspring had MDD.

Our findings on the increased risk of medical illness in relation to BW status are consistent with previously demonstrated links between LBW and medical illness such as cardiovascular illness [7] and diabetes [8]. The current study, however, examined a wider range of medical illnesses, including several illnesses related to HPA axis functionality (hypertension, respiratory illness, and allergies). We found that in offspring with LBW relative to those with high BW, not only was the risk of hypertension elevated but so was the risk of allergies. However, the risk of hypertension became only marginally significant after we adjusted for potential confounders. Posthoc analysis revealed that although the overall prevalence of hypertension in female and male

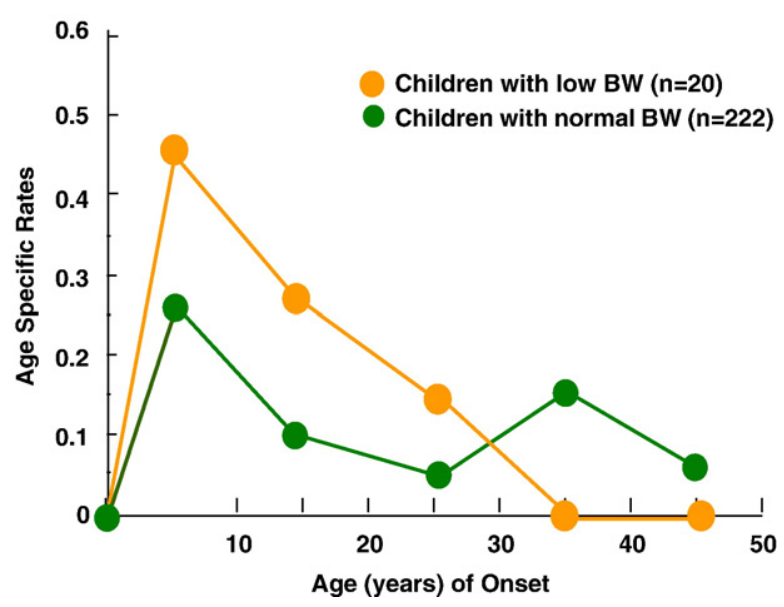

Fig. 2. Age-specific rates of anxiety disorder in children (second generation) by their low BW status.

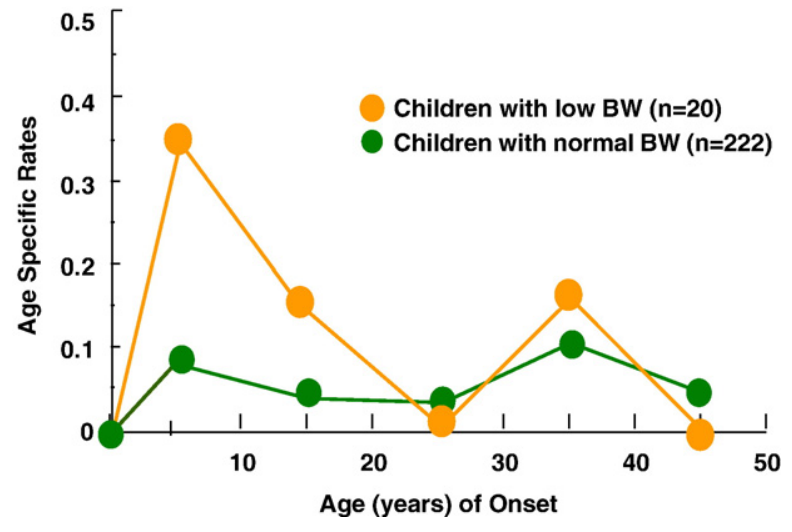

Fig. 3. Age-specific rates of any phobia in children (second generation) by their low BW status.

offspring was similar ( $4.7 \%$ vs $4.3 \%$ ), the risk of developing hypertension was much higher in male offspring with LBW $(\mathrm{RR}=16.7, P=.08)$ than in female offspring with LBW $(\mathrm{RR}=3.3, P=.38)$. Thus, when the sex effect was removed from the analysis, there was only a marginally increased risk of hypertension among offspring with LBW relative to those with high BW $(>3.5 \mathrm{~kg})$. Because the prevalence of medical illness in young adulthood is relatively low, the examination of a possible differential sex effect might be more reliable as offspring grow older. In sum, the risk of medical illnesses that were potentially related to HPA axis functionality appeared to be elevated in offspring with LBW relative to those with BW greater than $3.5 \mathrm{~kg}$, even in young adulthood. However, the magnitude of this association may not be fully appreciated until we follow the sample population into the ages of greater risk for these conditions.

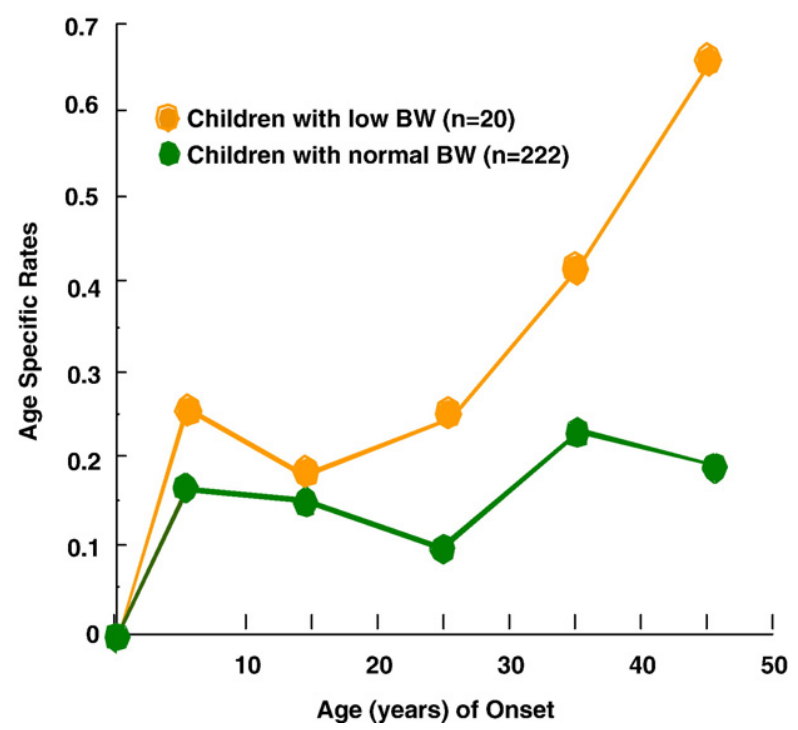

Fig. 4. Age-specific rates of medical illness in children (second generation) by their low BW status. 
As the magnitude of increased risk among offspring with LBW has public health implications, we had chosen to analyze the effect of BW by using a discrete category $(<2.5$, 2.5-3.5, and $>3.5 \mathrm{~kg}$ ) while recognizing that the risk of adult illness could increase in a dose-response manner as BW decreases. However, we also examined whether the data better fit a model in which the effects of BW were exerted dimensionally. The results showed that the risk was increased as BW decreased for $\operatorname{MDD}(P=.03)$, suicidal ideation $(P=.04)$, allergies $(P=.04)$, and functional impairment $(P=.01)$, with trends for asthma $(P=.09)$ and hypertension $(P=.10)$.

Results from related twin studies are consistent with our findings: twins with lower BW had higher psychologic distress [2], more behavioral problems [34], and a higher prevalence of psychopathology [35]. However, the hypothesized association between LBW and increased risk of depression or emotional distress in adolescence and adulthood in community-based studies $[4,10,12]$ is still inconclusive. Although Thompson et al [12] reported the association between LBW and depression only in men, another study did so only in women [10]. We found that both men (RR, 2.7; 95\% CI, 0.95-7.2) and women (RR, 2.7; 95\% CI, 1.2-5.8) who were born with LBW were at increased risk of MDD. The interaction between BW status and sex was not significant $(P=.96)$, indicating that LBW status increased the risk of MDD equally by sex in this sample.

One recent study [36] followed a cohort of 10753 male singletons from age 15 to 49 years for more than 30 years and reported no evidence for increased risk of depression by LBW. Their findings were based on hospital discharge records. Indeed, in our sample, among those who met the criteria for MDD, only 33\% received treatment and only $13 \%$ were ever hospitalized for their psychiatric condition. Those who were hospitalized, relative to those who were not, may have had more severe forms of depression with different causal pathways.

Taking advantage of the high-risk study design, we examined separately the impact of LBW on the risk of psychiatric and medical illnesses among offspring of depressed and nondepressed parents. This is not possible with other community-based studies on the prevalence of depression among adolescents and adults born with low or normal BW. We hypothesized that the adverse impact of LBW on psychopathology would be stronger among offspring of depressed, relative to nondepressed, parents. Analyses stratified by parental depression did appear to suggest that risks for all illnesses, except the respiratory illness, were greater among offspring of depressed relative to offspring of nondepressed. However, a formal test of interaction between parental depression and BW status was not significant for most disorders except depression and impaired functioning (only marginally significant). Unfortunately, the number of subjects, especially in the group with LBW and nondepressed parents, may have been too small to detect an interaction for many of the disorders. In some twin studies, the interaction of familial history of psychopathology and BW status has been tested. One study found no evidence for a synergistic interaction of $\mathrm{BW}$ and parent psychopathology on child psychopathology [34], but other studies reported an antagonistic interaction, in which children with LBW (adjusted for gestational age), compared to those with normal $\mathrm{BW}$, were less sensitive to familial or genetic effects on increased risk of behavioral problems [37] and cognitive development [38]. Twin studies have a methodologic strength over community and/or epidemiologic studies in controlling for extraneous effects. However, none of the mentioned twin studies used DSM-IV diagnoses. Future studies, including those conducted on twins, could benefit from application of the Diagnostic and Statistical Manual of Mental Disorders (DSM)-IV diagnostic criteria.

In our study, offspring with LBW showed a higher incidence of psychiatric disorders in their early years (5-15 years) relative to those with normal BW (mid and high BW). The occurrence of medical illnesses was more prominent after childhood and adolescence. In our sample, because of the high-risk design, the prevalence of affective disorders was higher than that found in community samples, which permitted documentation of these disorders throughout the life cycle. However, among the medical illnesses we studied, with the exception of allergies, prevalence rates were relatively low. Given the low prevalence rates of medical illnesses, the clear difference in age-specific rates in offspring in later life by BW is noteworthy.

Previous studies suggest that stressful prenatal events associated with maternal smoking, substance use, preterm birth, and LBW may contribute to abnormalities in brain morphology and function (cognition, emotionality, and behavior), and may compromise the integrity of the endocrine and immune system [15]. One possible mechanism that could explain the susceptibility to physical and psychiatric illness throughout the life span in infants with LBW is alteration in HPA axis activity and reactivity. To determine whether this is a reasonable hypothesis to pursue in more focused research investigations, we conducted secondary data analyses in a longitudinal study originally designed to understand the intergenerational transmission of MDD. We examined the risk for both physical and psychiatric illnesses known to be influenced by HPA axis dysregulation. Our findings provided preliminary evidence of increased risk for both physical and psychiatric illnesses that are associated with HPA axis functioning. Incorporating direct measures of HPA functionality into future studies could significantly enhance our ability to confirm the hypothesis that increased adult illness among offspring with LBW is mediated by HPA axis dysregulation. This longitudinal study of intergenerational transmission of MDD provides the preliminary evidence to validate further studies into this link.

Although we have considered maternal risk behavior, such as smoking, drinking, and substance use during pregnancy as confounders, we also examined the extent to 
which the risk behavior during pregnancy was associated with psychiatric and medical illness outcomes in offspring, mediated through BW status. Although a previous study in this sample demonstrated that maternal smoking during pregnancy increased risk for conduct disorder and attention deficit hyperactivity disorder in their offspring [39]. The results show that smoking was associated with an increased risk for LBW, but was not associated with any of the offspring outcomes under examination. In sum, although LBW represented an increased risk for affective and anxiety disorders (psychiatric illness) and medical illnesses related to reactivity (respiratory, allergies, and hypertension), maternal risk behavior during pregnancy, such as smoking, was not directly associated with the risk of these illnesses.

\subsection{Strengths and limitations}

This study bears the following strengths: diagnoses based on standardized, in-depth psychiatric interviews with bestestimate procedures; a very long follow-up time (20 years) affording examination of age-specific incidence rates by BW status; and analyses based on proportional hazards models rather than logistic regression, permitting variable follow-up times. In using a high-risk sample, we could maximize the potential case yield for affective disorders, minimize heterogeneity, and identify early patterns of illness, while evaluating whether parental depression status moderated the relationship between BW and offspring disorders.

However, the study also has some limitations. First, the number of LBW offspring is relatively small, so results should be interpreted cautiously. Because of this limitation, we were unable to divide the LBW further into LBW (2.5$1.5 \mathrm{~kg})$ and very LBW $(<1.5 \mathrm{~kg})$ groups, although we divided normal BW into 2 groups $(2.5-3.5$ and $>3.5 \mathrm{~kg})$ and examined a gradient relationship across the BW range on offspring disorders. Second, we found partial evidence to support an interaction between parental depression and LBW on offspring illness outcomes (MDD $[P=.05]$ and impaired functioning $[P=.098])$. The absence of statistically significant interactions between LBW and parental depression on other disorders, however, has to be interpreted cautiously because the small number of LBW cases in offspring of nondepressed parents may have limited the statistical power to examine the significance of interactions. Third, the measure of BW relied on parents' retrospective reports. Although several studies reported that BW reported by mothers was valid [40], it may be subject to recall bias. We have multiple reports on BW with excellent reliability $(r$ $=.87$ ). In addition, 66 offspring now have their own children and reported their BW at both wave 3 and 4, which also showed high reliability $(r=.94)$. Taken together, it is reasonable to assume that parental reports of BW are fairly accurate in our sample. Finally, results are based on offspring of depressed parents who sought treatment of their depression as well as nondepressed age- and sex-matched control, not a community sample. Thus, generalizability may be limited. However, unlike other community-based studies, diagnoses were based on rigorous best-estimate procedure deriving from the DSM-IV criteria; and medical illness status was updated through interviews and medical records. Any discrepancy was consolidated by independent assessors blind to parental depression and BW status. Despite the small sample size, our clinical data show a future direction of the investigation in association between LBW and psychiatric and medical illnesses across life. Future communitybased studies could benefit from application of the DSM-IV diagnostic criteria.

\section{Acknowledgment}

We appreciate the helpful comments of Virginia Warner on the earlier version of the manuscript.

\section{References}

[1] Gray RF, Indurkhya A, McCormick MC. Prevalence, stability, and predictors of clinically significant behavior problems in low birth weight children at 3, 5, and 8 years of age. Pediatrics 2004;114:932-40.

[2] Cheung YB, Ma S, Machin D, et al. Birthweight and psychological distress in adult twins: a longitudinal study. Acta Paediatr 2004;93:965-8.

[3] Bhutta AT, Cleves MA, Casey PH, et al. Cognitive and behavioral outcome of school-aged children who were born pre-term: a metaanalysis. JAMA 2002;288:728-37.

[4] Saigal S, Pinelli J, Hoult L, et al. Psychopathology and social competencies of adolescents who were extremely low birth weight. Pediatrics 2003;111:969-75.

[5] Rickards AI, Kelly EA, Doyle LW, et al. Cognition, academic progress, behavior and self-concept at 14 years of very low birth weight children. J Dev Behav Pediatr 2001;22:11-8.

[6] Barker DJ. The developmental origins of chronic adult disease. Acta Paediatr Suppl 2004;93:26-33.

[7] Leon DA, Lithell HO, Vagero D, et al. Reduced fetal growth rate and increased risk of death from ischaemic heart disease: cohort study of 15000 Swedish men and women born 1915-1929. BMJ 1998;317: 241-5.

[8] Lithell HO, McKeigue PM, Berglund L, et al. Relation of size and birth to non-insulin dependent diabetes and insulin concentrations in men aged 50-60 years. BMJ 1996;312:406-10.

[9] Indredavik MS, Vik T, Heyerdahl S, et al. Psychiatric symptoms and disorders in adolescents with low birth weight. Arch Dis Child Fetal Neonatal Ed 2004;89:F445-50.

[10] Gale CR, Martyn CN. Birth weight and later risk of depression in a national birth cohort. Br J Psychiatry 2004;184:28-33.

[11] Wiles N, Peters TJ, Leon DA, et al. Birth weight and psychological distress at age 45-51 years. Br J Psychiatry 2005;187:21-8.

[12] Thompson C, Syddall H, Rodin I, et al. Birth weight and the risk of depressive disorder in later life. Br J Psychiatry 2001;179:450-5.

[13] Wadhwa PD. Psychoneuroendocrine processes in human pregnancy influence fetal development and health. Psychoneuroendocrinology 2005;30:724-43.

[14] Arborelius L, Owens MJ, Plotsky PM, Nemeroff CB. The role of corticotropin-releasing factor in depression and anxiety disorders. J Endocrinology 1999;160:1-12.

[15] Heim C, Nemeroff CB. The role of childhood trauma in the neurobiology of mood and anxiety disorders: preclinical and clinical studies. Biol Psychiatry 2001;49:1023-39.

[16] McEwen BS. Protective and damaging effects of stress mediators. N Engl J Med 1998;338:171-9. 
[17] Nomura Y, Brooks-Gunn J, Davey C, et al. The role of perinatal problems for risk of comorbid psychiatric and medical disorders in adulthood. Psychol Med [in press].

[18] Nomura Y, Wickramaratne P, Warner V, et al. Family discord, parental depression and psychopathology in offspring: 10-year follow-up. J Am Acad Child Adolesc Psychiatry 2002;41:402-9.

[19] Weissman MM, Gammon GD, John K, et al. Children of depressed parents: increased psychopathology and early onset of major depression. Arch Gen Psychiatry 1987;44:847-53.

[20] Weissman MM, Fendrich M, Warner V, et al. Incidence of psychiatric disorder in offspring at high and low risk for depression. J Am Acad Child Adolesc Psychiatry 1992;31:640-8.

[21] Weissman MM, Warner V, Wickramaratne P, et al. Offspring of depressed parents: 10 years later. Arch Gen Psychiatry 1997;54:932-40.

[22] Weissman MM, Wickramaratne P, Nomura Y, et al. Families at high and low risk for depression: a 3-generation study. Arch Gen Psychiatry 2005;62:9-36

[23] Mannuzza S, Fyer AJ, Klein DF, et al. Schedule for affective disorders and schizophrenia-lifetime version modified for the study of anxiety disorders (SADS-LA): rationale and conceptual development. J Psychiatr Res 1986;20:317-25.

[24] Orvaschel H, Puig-Antich J, Chambers W, et al. Retrospective assessments of prepubertal major depression with the Kiddie-SADSE. J Am Acad Child Adolesc Psychiatry 1982;21:392-7.

[25] Endicott J, Spitzer RL, Fleiss JL, et al. The global assessment scale: a procedure for measuring overall severity of psychiatric disturbance. Arch Gen Psychiatry 1976;33:766-71.

[26] Shaffer D, Gould MS, Brasic J, et al. Children's global assessment scale (C-GAS). Arch Gen Psychiatry 1983;40:1228-31.

[27] Leckman JF, Sholomskas D, Thompson D, et al. Best estimate of lifetime psychiatric diagnosis: a methodological study. Arch Gen Psychiatry 1982;39:879-83.

[28] Hollingshead AB, Redlich FC. Social class and mental illness. New York (NY): John Wiley; 1958.
[29] Cox DR. Regression models and life tables. Appl Stat 1972;34: $87-220$.

[30] Weissman MM, Warner V, Fendrich M. Applying impairment criteria to children's psychiatry diagnosis. J Am Acad Child Adolesc Psychiatry 1990;29:789-95.

[31] Binder DA. Fitting Cox's proportional hazards models from survey data. Biometrika 1992;79:139-47.

[32] Lin DY, Wei LJ. The robust inference for the proportional hazards model. J Am Stat Assoc 1989;84:1074-8.

[33] Shah BV, Barnwell BG, Gayle GS. SUDAAN user's manual 7.5. Research Triangle Park (NC): Research Triangle Institute; 1997.

[34] Van Os J, Wichers M, Danckaerts M, et al. A prospective twin study of birth weight discordance and child problem behaviour. Biol Psychiatry 2001;50:593-9.

[35] Wals M, Reichart CG, Hillegers MH, et al. Impact of birth weight and genetic liability on psychopathology in children of bipolar parents. J Am Acad Child Adolesc Psychiatry 2003;42:1116-21.

[36] Osler M, Nordentoft M, Andersen AMN. Birth dimensions and risk of depression in adulthood: cohort study of Danish men born in 1953. Br J Psychiatry 2004;186:400-3.

[37] Wichers MC, Purcell S, Danckaerts M, et al. Prenatal life and postnatal psychopathology: evidence for negative gene-birth weight interaction. Psychol Med 2002;32:1165-74.

[38] Koeppen-Schomerus G, Eley TC, Wolke D, et al. The interaction of prematurity with genetic and environmental influences on cognitive development in twins. J Pediatr 2000;137:527-33.

[39] Weissman MM, Warner V, Wickramaratne PJ, et al. Maternal smoking during pregnancy and psychopathology in offspring followed to adulthood. J Am Acad Child Adolesc Psychiatry 1999;38:892-9.

[40] Olson JE, Shu XO, Ross JA, et al. Medical record validation of maternally reported birth characteristics and pregnancy-related events: a report from the Children's Cancer Group. Am J Epidemiol 1997;145: 58-67. 